\title{
Cardiovascular Aspects in Endocrine Diseases - Some Considerations Regarding this Dual and Vital Network Relationship
}

\author{
Stoia Mirela-Anca* \\ Department of Internal Medicine, Cardiology and Gastroenterology, University of Medicine and Pharmacy, Cluj-Napoca, Romania
}

Submission: February 16, 2018; Published: March 29, 2018

*Corresponding author: Stoia Mirela-Anca, Department of Internal Medicine, Cardiology and Gastroenterology, University of Medicine and Pharmacy, Department of Cardiology, County Clinical Emergency Hospital, Cluj-Napoca, Romania, Tel: 0040722280952,

Email: mirelastoia@yahoo.com

\section{Mini Review}

Normal endocrine function is essential for cardiovascular health. Disorders of the endocrine system, consisting of hormone hyperfunction or hypofunction, have multiple effects on the cardiovascular system [1]. We can summarize these aspects in relation with cardiovascular risk, cardiovascular hemodynamics changes, cardiac structure and function alteration, rhythm and cardiac conductance perturbations.

Cardiovascular risk (CVR) in endocrine disorders is associated first with metabolic imbalance and consists in increased body fat and central obesity, dyslipidemia (low high density lipoprotein cholesterol (HDLc) high total cholesterol, high low density lipoprotein cholesterol (LDLc)), increased lipoprotein (a), apolipoprotein B, reduced number of hepatic LDL receptors, high triglycerides, impaired fasting glucose or tolerance, hyperinsulinemia, decreased insulin sensitivity and diabetes mellitus [2-6].

The metabolic disorder are correlated with vascular morphology and function changes (impaired nitric oxide availability, endothelial dysfunction, altered flow-mediated dilatation, increased arterial stiffness, reduced arterial compliance, vascular hypertrophy, fibrosis and calcification, increased carotid arterial intima-media thickness (IMT), a marker of early atherosclerotic development and accelerated atherosclerosis). This pattern is related to some endocrine hypofunction (growth hormone deficiency- acromegaly (GHD), hypothyroidism) and some endocrine hyperfunction (hypercortisolism (Cushing's syndrome), hyperparathyroidism (PHPT), primary hyperaldosteronism (PA), pheochromocytoma). The addition of arterial hypertension (HT) in combination with increased preload and cardiac output, plasma volume expansion and increased arterial resistance raise the CVR. The presence of hyperviscosity (hyperhomocysteinemia), inflammation (increased C-reactive protein levels), altered coagulation parameters (stimulated endothelial production of von Wille brand factor and increased factor VIII), enhanced platelet aggregation and reduced plasma fibrinolytic potential augmented the incidence of cardiovascular [2-6].

Cardiac structure and function are modified in endocrine disorders. There are abnormalities including myocyte hypertrophy, interstitial fibrosis (collagen deposition, activation of inflammatory cells and stimulation of fibroblast proliferation), inflammatory cell infiltration, reduced capillary density, myofibril derangement and extracellular collagen deposition. All of them can lead to ischemic heart disease, cardiomyopathy with left ventricular (LV) cavity dilatation (or biventricular), diastolic and systolic dysfunction and heart failure [2-6].

Echocardiography reveals LV hypertrophy (LVH), more often concentric type (sometime irrespective to the presence of HT), with significant reductions in LV posterior wall thickness and interventricular septal thickness, with resultant decreases in $\mathrm{LV}$ internal diameter, and increases in LV mass index, impaired LV end diastolic volume (LVEDV), overload and stroke volume, reduced ejection fraction, left atrial dilatation. Most of patients with endocrine disorders have impaired LV performance at peak exercise, and report exercise intolerance. In the early stage of hyperfunction endocrine disorders, there is enhanced myocardial contractility, decreased systemic vascular resistance, increased cardiac output, and overall increased cardiac performance (compensatory mechanism and compensated stage). The hypofunction endocrine disease can be associated with abnormal cardiac infiltration (edema, collagen, amyloid, glycogen, calcium, catecholamines). The endocrine disease progression produces the enlargement of cavity and the thinning of walls, impairment diastolic and systolic functions appears and enhanced pulmonary and wedge pressures. Diastolic dysfunction has been demonstrated with impaired early LV filling and relaxation, longer isovolumetric relaxation times, and evidence of global myocardial relaxation impairment. In advance stages of endocrine disorders we assisted to such 
called maladaptive cardiac remodeling. The completion between vascular and myocardial stiffness is related to an even worse cardiovascular state, the presence o heart failure demonstrated a poor prognostic. The electrolytes imbalance can lead to impaired excitation-contraction coupling and supplementary impairing of the systolic function, but also can be responsible of arrhythmias. The valvular endocardium (mitral, aortic) can be affected by myxomatous, fibrosis and calcific process related with regurgitation or stenotic hemodynamics [2-6].

Electrocardiogram (ECG) and holter studies have documented cardiac rhythm abnormalities in various endocrine disorders. In endocrine hyperfunctions, resting ECG changes include left (or combined right) axis deviation, increased QRS complex amplitude, shortened QT intervals, septal Q-waves, ST segment depression, inverted or biphasic T waves and late potentials that could predispose to arrhythmias. Rhythm disturbances, seen mainly during physical exercise, include sinus tachycardia, atrial and ventricular ectopic beats, paroxysmal atrial fibrillation, paroxysmal supraventricular tachycardia, sick sinus syndrome, bundle branch block, and ventricular tachycardia. The frequency of ventricular premature complexes increases with the duration of endocrine disorders and the severity of ventricular arrhythmias correlates with increases in LV mass. The factors independently predictive of atrial fibrillation include increasing age, history of cardiac failure, diabetes, HT and LVH on ECG and increased age and duration of atrial fibrillation correspond with higher rates of persistent arrhythmia. At the opposite side, in endocrine hypofunctions, include sinus bradycardia, low voltage complexes (small P waves or QRS complexes), prolonged PR and QT intervals and flattened or inverted $\mathrm{T}$ waves, ventricular conduction abnormalities may be related to QT interval prolongation [2-6].

The majority of the cardiovascular diseases development, especially complicated with heart failure and with the presence of metabolic syndrome and HT are linked to the onset and the imbalance of multiple neuro-endocrine and other regulation systems. The most important are renin-angiotensin-aldosterone, adrenergic and calcium-calmodulin contraction systems. All of them are implicated in endocrine disorders too $[7,8]$. In many cases, despite the "classic" scenario, the cardiovascular symptoms (palpitations due to arrhythmias, angina pectoris, and dyspnea related to heart failure) can be one of the first alarm signal for the presence of an "hidden" endocrine disorder.

\section{References}

1. Baxter JD, Young WF, Webb P (2003) Cardiovascular Endocrinology: introduction. Endocr Rev 24(3): 253-260.

2. Rhee SS, Pearce EN (2011) The Endocrine System and the Heart: A Review. Rev Esp Cardiol 64(3): 220-231.

3. Bloomgarden ZT (2005) Endocrine Aspects of Cardiovascular Disease. Diabetes, Hypertension, and Cardiovascular Disease.

4. Stultz D (2008) Endocrine Disease and the Heart. Southwest Cardiology.

5. Ibarra C, Lavandero S, Strada ME (2015) Regulation of Cardiovascular Metabolism by Hormones and Growth Factors.

6. Sophie Mavrogeni (2009) Cardiac complications in endocrine diseases. European Society of Cardiology 7(26).

7. Ogawa T, de Bold AJ (2014) The heart as an endocrine organ. Endocrine Connection 3(2): R31- R44

8. Horvath K (2016) Matters of the Heart: The Vital Link between the Cardiovascular and Endocrine Systems. Endocrine News. Endocrine Society. 